\title{
Film VerORTen: Film als Forschungs- und Kommunikationsmedium in der Geographie
}

\author{
Susan Thieme, Philipp Eyer, and Alexander Vorbrugg \\ Geographisches Institut, Universität Bern, 3012 Bern, Switzerland \\ Correspondence: Susan Thieme (susan.thieme@giub.unibe.ch)
}

Received: 3 July 2019 - Revised: 17 October 2019 - Accepted: 21 October 2019 - Published: 22 November 2019

\begin{abstract}
Kurzfassung. In this "positioning" we discuss current developments, possibilities and challenges around working with film in and for geography. We describe possibilities that certain conscious and collaborative ways of employing film offer: They point beyond film analysis and are more than a mere add-on to communicate research results, but rather can stimulate new forms of reflexivity and creativity along different steps of research and teaching processes. We further show how the emergence of new digital and physical platforms can enable and support exchange on film and other digital media, using the example of our new media laboratory (mLab) at the University of Bern.
\end{abstract}

\section{Immer wieder neu: Schnittstellen zwischen Geographie und Film}

Vor über sechs Jahrzenten schrieb Georg Pool in der Geographica Helvetica über den Einsatz von Film im Geographieunterricht: „Der Unterrichtsfilm ist heute kein Unbekannter mehr. Seit seiner Einführung als Hilfsmittel des modernen Unterrichtes sind mehr als 30 Jahre vergangen. Dennoch ist bis heute die Diskussion über den Wert des Unterrichtsfilmes nicht abgeschlossen" (Pool, 1953). Pool ruft uns damit die nun beinahe hundertjährige Auseinandersetzung mit Film als Medium der Vermittlung geographischen Wissens im deutschsprachigen Raum in Erinnerung. In anderen Disziplinen, wie beispielsweise der Sozialanthropologie oder auch in weiten Teilen der anglophonen Sozialwissenschaften, wurde Film noch früher als Medium der Produktion und Kommunikation von Forschungsinhalten etabliert. Dennoch: Gegenwärtig und mit Blick in die Zukunft scheint uns die Diskussion über den Wert des Films aktueller und bedeutender denn je.

Vor dem Hintergrund möchten wir in diesem „Standpunkt“ aktuelle Entwicklungen, Herausforderungen und Möglichkeiten rund um den Film in der und für die Geographie erörtern und zu einer breiteren Diskussion in unserem Fach einladen. Insbesondere geht es uns um Möglichkeiten, Film in Formen einzusetzen, die sowohl über Filmanalysen als auch den Forschungsfilm als blosses Mittel der Ergebnisver- mittlung hinausgehen. Wir fokussieren dabei vor allem auf (1) die Potentiale von Filmproduktion für kollaborative Forschungsprozesse, (2) die Entstehung neuer Plattformen der Vermittlung, Interaktion und des Austauschs, bei denen Film im Mittelpunkt steht und (3) Erfahrungen aus der Lehre in der Arbeit mit Film. Zunächst umreissen wir aber aktuelle Entwicklungen rund um das Medium.

\section{Bedeutung von Film für die heutige Geographie}

Bildbasierte Medien haben rasant an Bedeutung gewonnen. Im Jahr 2017 machten Videos $70 \%$ des Umfangs der globalen Datenübertragung im Internet aus. Bis zum Jahr 2022 soll dieses Volumen um das Vierfache anwachsen und der Anteil dann ca. $80 \%$ des globalen Datenverkehrs betragen (CISCO, 2019). Gleichzeitig besteht gegenüber der Wissenschaft und damit auch der Geographie die steigende Erwartung, Wissen und Ergebnisse breitenwirksam zu kommunizieren und zur Diskussion zu stellen. Dies wird im Rahmen von Forschungsprogrammen und -förderungen, wie etwa denen des Schweizerischen Nationalfonds (SNF) und der EU oder auch der nationalen „Strategie Digitale Schweiz“, zunehmend explizit gefordert. Dabei werden Film und Video als besonders geeignete Mittel beschrieben, den Ertrag der Wissenschaften öffentlich sichtbar und zugänglich zu machen. Die Kommunikation von Forschungsinhalten in Fachzeitschriften und 
durch Text reicht dieser Logik nach nicht mehr aus und so werden Wissenschaftler*innen angehalten, die Möglichkeiten anderer Medien aktiv zu nutzen (Jacobs, 2013, 2016; Angelone, 2019). Schliesslich werden zunehmend auch Schritte zur Integration digitaler Elemente in die Hochschullehre gefordert, die häufig gezielt Lehrvideos einsetzen, wie beispielsweise Inverted Classroom oder Massive Open Online Courses (MOOC). Solche Entwicklungen können wir leicht, und aus unserer Sicht manchmal nicht ganz zu Unrecht, als eher unwillkommene Zwänge einer beschleunigten Informationsgesellschaft ansehen. Statt solche problematischen Seiten zu diskutieren, wollen wir uns an dieser Stelle aber für eine aktive Aneignung der Potentiale von Filmproduktion aussprechen. Dies ist umso wichtiger, je mehr wir Gefahr laufen, von externen Entwicklungen getrieben zu sein.

International entstehen gegenwärtig sehr vielfältige Projekte und Plattformen, die Wissenschaft, Öffentlichkeit und Film einander in unterschiedlichen Weisen näherbringen. Wissenschaftliche Filmfestivals mit und ohne Peer-ReviewVerfahren etablieren sich, bilden internationale Ableger oder werden neu ins Leben gerufen (z.B. Annals of Crosscuts, Imagine Science Film Festival). Parallel entstehen OnlineJournals für wissenschaftliche Filme wie das JVE (2019), ein peer reviewed Online-Journal für ethnographische Filme, oder die Plattform Labocine (Labocine, 2019). Auch in der Geographie entstehen neue Online-Plattformen für wissenschaftliche Filme (z.B. FilmGeographies, 2019) und mit der Film-Making and Screening Specialty Group gibt es seit Ende 2018 eine Institutionalisierung der wissenschaftlichen Filmproduktion unter dem Dach der American Association of Geographers (AAG, 2019). Das Global Science Film Festival bringt seit 2017 in Zürich und ab November 2019 auch in Bern das Genre Film, Wissenschaftler*innen unterschiedlicher Disziplinen und eine breitere städtische Öffentlichkeit auf neue Weisen zusammen (Global Science Film Festival, 2019). Um Möglichkeiten, solche Impulse in unseren Instituten aufzugreifen, soll es im folgenden Abschnitt gehen.

\section{Mit Film Brücken bauen und zusammenarbeiten}

Grundsätzlich lassen sich unterschiedliche Einsatzweisen von Film in den Sozialwissenschaften unterscheiden (Garrett, 2011), die in der deutschsprachigen Geographie jeweils unterschiedlich lange und prominent vertreten werden. So hat im Zuge des ,cultural turn“ und der Zuwendung zu visuellen Geographien (Schlottmann und Miggelbrink, 2009) vor allem die Filmanalyse Einzug in den etablierten Forschungsbestand erhalten (früh bspw. Escher und Zimmermann, 2001). In jüngerer Zeit hat sich auch die Produktion von Filmen zur Kommunikation von Forschungsinhalten weiter etabliert. Als integraler Bestandteil des Forschungsprozesses dagegen bleibt das Filmen in der deutschsprachigen Geographie bisher eher selten und hat in den letzten Jahren in der englischsprachigen Geographie viel mehr Beach- tung gefunden (zum Beispiel Baptiste, 2015; Garrett, 2011; Jacobs, 2016).

Dabei kommt gerade bei der Produktion von Filmen das Potential des Mediums besonders zur Geltung (Angelone, 2019; Thieme, 2012). So lässt sich das Filmemachen auf Weisen in Forschungsprozesse integrieren, die den Prozess des Forschens sowie der Filmproduktion sichtbar machen. In Teilen der Sozialanthropologie und der englischsprachigen Geographie wird dies im Rahmen qualitativer Feldforschungsprojekte sowohl zum Zweck der Dokumentation als auch im Sinne einer reflexiven oder partizipativen Forschungspraxis eingesetzt (Berardi und Mistry, 2012; Brickell und Garret, 2015; Kindon, 2003, 2016; Parr, 2007; Pink, 2007). Aber auch in Teilen der Naturwissenschaften kommt das Filmen zur Dokumentation, Reflexion und Vermittlung von Forschungsprozessen zum Einsatz (Pasquali, 2007). Film kann Trugbilder einer objektiven Realitätsvermittlung schaffen, das Filmemachen kann aber auch in Weisen eingesetzt werden, die den Entstehungsprozess des Films, also das ,making of“, als machtgeladenen, umkämpften und mitunter problembehafteten Prozess darstellen und zur Sprache bringen (Kindon, 2003, 2016; Brickell und Garret, 2015). Die Arbeit mit der Kamera erfordert und ermöglicht häufig mehr Interaktion, sowohl mit den Protagonist*innen als auch innerhalb des Filmteams. Vorgehensweisen, Inhalte und Verantwortlichkeiten während des Filmprozesses müssen diskutiert und transparent gemacht werden (Thieme, 2012). Die Arbeit mit der Kamera kann als Mittel gemeinsamer Reflexion und Aushandlung eingesetzt und der Blick auch gezielt auf die Filmenden gerichtet werden. Digitalkameras bieten den Involvierten die Möglichkeit, das unbearbeitete Material sofort einsehen und kommentieren zu können. Aufgrund der Synchronität von Visuellem, Sensorischem und Performativem (Barbash-Taylor, 1997; Ambühl, 2018) ist Filmmaterial selbst in der Rohform für viele Menschen schneller zugänglich als Daten in Textform, wie beispielsweise transkribierte Interviews (Angelone, 2019). Zudem können Themen von Macht und Repräsentation im Kreis der Beteiligten analysiert werden. Wer wirkt mit am Film? Wie wird die Rollenverteilung zwischen ,Repräsentierenden“ und „Repräsentierten“ ausgehandelt und aufgebrochen? Wem kommt der Film zugute? Wie produziert oder destabilisiert ein Film Wahrheitsansprüche? Wie werden das bewegte Bild und der gesprochene oder eingeblendete Text in Beziehung zueinander gesetzt? Was wird gefilmt, was nicht? Wer entscheidet was im Film sicht- und hörbar wird und was nicht? Beim Zeigen des fertigen Films können die Mitwirkenden eingeladen werden. Dabei entstehen Begegnungen zwischen den Forschenden, Protagonist*innen und $\mathrm{Pu}-$ blikum, wobei die Filmschaffenden den Film mit dem $\mathrm{Pu}-$ blikum diskutieren und neue Forschungsfragen und Filmideen daraus generieren können. Zudem bietet Film die Möglichkeit, durch Untertitel in mehrere Sprachen übersetzt und leicht online zugänglich gemacht zu werden. Verglichen mit vielen anderen Formaten der Wissenschaftskommunikation 
lassen sich hier also manche Barrieren der weiten Vermittlung effektiv überwinden.

\section{Orte der Kollaboration schaffen: Medienlabore in der Geographie}

Dass die Geographie als integratives Fach eine Schnittstellenfunktion zwischen gesellschafts- und geisteswissenschaftlichen Ansätzen einerseits und naturwissenschaftlichen andererseits wahrnehmen sollte, wird immer wieder gefordert (Schlottmann et al., 2018). Die Umsetzung dieses Anspruchs scheitert in der Praxis aber gar nicht selten an der Unterschiedlichkeit der Fachkulturen, Konzepte und Wissenschaftslogiken und so herrscht oft doch Sprachlosigkeit, wo eigentlich Austausch und Zusammenarbeit gefordert werden. Unserer Erfahrung nach liegt die Schwierigkeit oft weniger in einem Mangel geteilter Anliegen oder Themen, sondern mehr darin, eine gemeinsame Sprache zu entwickeln, um diese Themen zu adressieren. Gerade hier bietet die gemeinsame Filmproduktion wichtige Möglichkeiten für integrative Forschungsprojekte und Vermittlungsformen.

Für dieses alternative und kreative Denken braucht es auch physische Räume. An verschiedenen Universitäten gab und gibt es Initiativen, Orte einzurichten, an denen mit Film und anderen Medien gearbeitet werden kann Medien gearbeitet werden kann, so zum Beispiel in Genf (Fall, 2019), Kiel (GeoMedien, 2019) und Vancouver (Emerging Media Lab, 2019). Auch am Geographischen Institut der Universität Bern sind wir seit zwei Jahren dabei, ein Medienlabor aufzubauen. In Anlehnung an die in den Naturwissenschaften etablierte Idee eines Labors dient das Medienlabor (mLab, 2019) als Ort, an dem nicht nur die technische Infrastruktur zur Verfügung steht. Vielmehr ist es ein Coworking Space, ein Ort des Austauschs, an dem mit einem methodischen Fokus fachspezifisch und interdisziplinär geforscht, gelernt und gelehrt wird. Der Erstellungsprozess eines Filmes ist häufig kollaborativ und interaktiv. Die Bereitstellung eines Raums fördert die Interaktion zwischen Studierenden, aber auch zwischen Studierenden und Dozierenden. Das Labor wird damit zu einem Raum der Kommunikation und Kollaboration. An unserem Institut hat die Etablierung des mLabs bereits zu enger Zusammenarbeit und Co-Leitung des mLabs mit einer weiteren Forschungsgruppe (Truong und Schurr, 2019) geführt und Instituts- und Fakultätsinitiativen zu einer breiteren Auseinandersetzung mit Digitalisierung und Medien angestossen. Eine gemeinsame Mitteleinwerbung auf $\mathrm{Fa}$ kultätsebene erlaubt nun einen personellen Ausbau des mLabs, internationale Kolloquien zu Themen rund um Digitalisierung, eine gemeinsame Durchführung des Global Science Filmfestivals mit Zürich und kurzzeitige Finanzierungen für Initiativen, durch die die Zusammenarbeit von Wissenschaftler*innen und Kunstschaffenden gefördert wird. Zudem ist das mLab keineswegs allein auf Film fokussiert, sondern versteht sich als Ort und Netzwerk, an dem mit und zu digitalen
Medien mit verschiedenen geographischen Perspektiven kritisch und kollaborativ gearbeitet wird.

\section{Film und Geographie in der Lehre}

Als Schnittstelle zwischen naturwissenschaftlich und sozialund geisteswissenschaftlich ausgerichteten Subdisziplinen wird in der Geographie methodisch sehr breit ausgebildet. Umso wichtiger erscheint es, in die Ausbildung der Studierenden technologiegestütztes Lernen und Forschen einzubauen, das natürlich nicht nur Film beinhaltet, sondern weit darüber hinaus geht. Dies ist neben der Erfahrung des kollaborativen Arbeitens auch eine wichtige Erfahrung für den späteren Berufsalltag, den die wenigsten der Studierenden in einem universitären Umfeld bestreiten werden (Angelone, 2019). Gerade Studierende, die im Alltag oft Medien nutzen, erleben in der Lehre eher selten (abgesehen vom Abspielen von Videos), dass Film mit konkreter wissenschaftlicher Praxis verbunden wird und damit Inspiration liefert, wie sie fachliches Wissen an der Universität und später in der Berufspraxis praktisch umsetzen und filmisch kommunizieren können.

Neue und oft auch forschungsgeleitete Lehrideen im $\mathrm{Zu}$ sammenhang mit dem mLab führten bisher zur Zusammenarbeit mit anderen Fakultäten und Partnern aus der Praxis, wofür wir auch universitäre Förderung für die Einführung filmbasierter Kurse erhielten. So zum Beispiel ein Seminar, in dem die Studierenden einen transdisziplinären Forschungsprozess durchlaufen und mit der Social-LearningVideo-Methode erarbeiten, wie ein gemeinsamer Lernprozess zwischen Forschung und Akteuren aus anderen Gesellschaftsbereichen angeregt werden kann und wo die Grenzen und Möglichkeiten der Methode liegen. Hier diskutieren wir auch explizit die disziplinäre Schnittstellenfunktion der Geographie im Zuge der Nachhaltigkeitsdebatten und das Potential von Transdisziplinarität im Sinne einer Koproduktion von Lösungen durch Wissenschaft, Zivilgesellschaft, Verwaltung und Wirtschaft (Fry und Thieme, 2019).

Im Kurs „Film und Geographie“ bekommen Studierende eine Einführung ins Filmemachen und erarbeiten einen dokumentarischen Kurzfilm mit einer spezifischen geographischen Fragestellung. Dabei stehen die kritische Reflexion des Filmprozesses und die Potentiale und Grenzen der unterschiedlichen filmbasierten methodischen Zugänge innerhalb wissenschaftlicher Forschung im Vordergrund. Das anschliessende gemeinsame Schauen der Kurzfilme bei einem Lunchkino, offen für das ganze Institut, regt wiederum den Austausch unterschiedlicher Perspektiven unter dem Dach der Geographie an. Über Film hinaus werden auch in anderen Lehrveranstaltungen Formate wie Podcasts, Videoabstracts oder auch Massive Open Online Kurse nicht nur stärker eingesetzt, sondern auch konzeptionell gerahmt und kritisch reflektiert. In einem weiteren Kurs lernen interessierte Studierende beim Mitorganisieren des Global Science Film- 
festivals nun auch, wie eine Veranstaltung an der Schnittstelle zwischen Film und Forschung umgesetzt werden kann.

Grundsätzlich zeigen unsere Lehrerfahrungen, dass Studierende oft bereits vielfältigste alltägliche Erfahrung mit der Nutzung und auch Produktion von Videos mitbringen. In der ,Flut“ an Filmen erscheint der Entstehungsprozess eines Filmes aber häufig als „Black Box“. Hier bietet eine Erweiterung der methodischen Ausbildung den Studierenden die Möglichkeit, den Prozess der Erstellung und der Rezeption des Films transparent zu machen und für Reflexion zu öffnen. Die Arbeit mit der Kamera konfrontiert die Studierenden mit ähnlichen Fragen wie auch textbasierte Bachelorund Masterarbeiten. Fragen zur Produktion und Vermittlung von Wissen, zu Zugängen ins Feld, zur Rolle als Forschende, zur Repräsentation und zu Forschungsethik. Einen dokumentarischen Film mit einer wissenschaftlichen Fragestellung zu produzieren bietet den Studierenden die Möglichkeit, vielfältige Erfahrungen mit unterschiedlichen qualitativen Methoden gleichzeitig zu machen. Dabei erleben Studierende, was es heisst, kollaborativ zu arbeiten, wie es sich anfühlt, im Feld zu sein, wie man als Forschende*r wahrgenommen wird und welche Dynamiken sich in der Beziehung zu den Teilnehmenden entwickeln können. Die Kamera zeigt dabei nicht nur das ,,ausgewählt“ Beobachtete, sondern widerspiegelt auch die eigene Haltung als Forschende*r. Der ,,limitierte" Blick durch die Linse und der Entscheidungsdruck am Schnittpult bieten den Studierenden einen neuen kreativen Zugang, sich kritisch mit der qualitativen sozialwissenschaftlichen Konstruktion von Wissen auseinanderzusetzen.

Im Kontext einer beschleunigten Medien- und insbesondere Videoproduktion (s.o.), aber auch wissenschaftlichen $\mathrm{Pu}$ blikationstätigkeit (Halffman und Radder, 2015), erscheint die eigene Produktion von Film als Kontrast. Die einzelnen Phasen des Filmemachens zu durchlaufen ist aufwändig und zeitintensiv, gleichzeitig gibt es den Durchführenden aber auch die Möglichkeit, Perspektiven auf einen Gegenstand zu wechseln und Inhalte anders zu erzählen (Angelone, 2019). Zudem eröffnet es auch die Erfahrung, dass die Arbeit mit Film auf den ersten Blick häufig als ,etwas ganz Anderes“ oder als „sehr kreativ“ wahrgenommen wird. In der konkreten Umsetzung können dann aber neben dem Mehrwert der Arbeit mit Film auch die vielfältigen Überschneidungen mit anderen (sozialwissenschaftlichen) methodischen Herangehensweisen und die generelle Erkenntnis, dass auch eine filmische Arbeit genau wie andere Forschungsarbeiten sehr zeitintensiv ist, herausgestellt werden.

\section{Geographie durch Film mitgestalten}

Dieser Beitrag ist ein Plädoyer für eine aktive, selbstbestimmte und kritische Aneignung der Potentiale von Film für die Geographie - gerade in einer Zeit, in der sich die Auseinandersetzung mit Film und digitalen Medien allgemein manchmal fast unangenehm aufzudrängen scheint. Aktuell werden Forschungsergebnisse immer noch zu einem überwiegenden Teil in Textform verfasst. Trotz Diskussionen und Initiativen um kreative Wissensbildung und -vermittlung, wie die Etablierung neuer Zeitschriften, Kurse an Hochschulen, Ausstellungen und Filmfestivals, ist Film als integraler Bestandteil von Forschung und Lehre in der Geographie nur marginal präsent (Ambühl, 2018; Baptiste, 2015; Garrett, 2011; Jacobs, 2016). Deshalb haben wir Möglichkeiten aufgezeigt, wie auch die Geographie das Potential von Film mehr für sich nutzen kann.

Film macht durch visuelle, sensorische, akustische und performative Dimensionen die soziale Konstruktion von Orten und Identitäten erfahrbar - und damit auch geographische Konzepte für ein breites Publikum zugänglich. Es braucht jedoch Wissen darüber, wie man Film nicht nur als Repräsentations- und Kommunikationsinstrument einsetzt, sondern wie man gezielt Gesellschaft auch filmisch erforschen kann. Hier spielt eine wichtige Rolle, wie Räume sozial produziert, repräsentiert und wahrgenommen werden Fragen die durch eine räumliche Perspektive adressiert werden können. Konkret kann dies nur geschehen, indem Forschende und Studierende sich aktiv, selbstbestimmt, kollaborativ und kritisch die unterschiedlichen methodischen $\mathrm{Zu}$ gänge, die sich über das Medium Film (und anderer Medien) ergeben, aneignen und damit experimentieren, um die Geographie theoretisch, methodisch und empirisch weiterzuentwickeln und auch weiterhin als wichtige Fachdisziplin in der Gesellschaft zu verankern. Labore als physische Orte und virtuelle Plattformen bieten auf unterschiedliche Weisen genau hierfür eine Basis.

Datenverfüg barkeit. Für den Artikel wurden keinen eigenen empirischen Daten erhoben. Alle konsultierten Referenzen und Internetseiten sind im Literaturverzeichnis einsehbar.

Autorenmitwirkung. Alle Autor*innen in haben in gleichem Umfang zum Artikel beigetragen. Die Initiierung und Koordination des Artikels übernahm die Erstautorin.

Interessenkonflikt. Die Autoren erklären, dass kein Interessenkonflikt besteht.

Begutachtung. This paper was edited by Benedikt Korf and reviewed by one anonymous referee.

\section{Literatur}

AAG: Film-making and Screening Specialty Group, online aufrufbar: http://www.aag.org/cs/membership/specialty_groups, letzter Zugriff: 11 November 2019. 
Ambühl, H.: Filmmaking Geography: Eine kreativ-transdisziplinäre Forschungs- und Kommunikationsmethode für die Geografie, Masterarbeit, Universität Bern, 2018.

Angelone, S.: A New Generation of Scientists-as-Filmmakers: Experiences Gained in Switzerland, Science Communication, 30, 5809, https://doi.org/10.1177/1075547019837620, 2019.

Annals of Crosscuts 2019: online aufrufbar: https://www.kth.se/ en/abe/inst/philhist/historia/ehl/ehl-events/crosscuts-film-festi/ annals-of-crosscuts, letzter Zugriff: 11 November 2019.

Baptiste, A. K.: Can a research film be considered a stand-alone academic publication? An assessment of the film Climate Change, Voices of the Vulnerable, The Fishers' Plight, 2015.

Barbash, I. and Taylor L.: Cross-cultural filmmaking: A handbook for making documentary and ethnographic films and videos, Berkeley, Los Angeles, London, University of California Press, 1997.

Berardi, A. and Mistry, J.: The challenges and opportunities of par video in geographical research: exploring collaboration with indigenous commun the North Rupununi Guyana, Area, 44, 110 116, 2012

Brickell, K. and Garrett, B. L.: Participatory politics of partnership: video workshops on domestic violence in Cambodia, Area, 47, 230-236, 2015.

CISCO (Cisco Visual Networking Index): Forecast and Trends, 2017-2022, online aufrufbar: https://www. cisco.com/c/en/us/solutions/collateral/service-provider/ visual-networking-index-vni/white-paper-c11-741490.pdf, letzter Zugriff: 11 November 2019.

Escher, A. and Zimmermann, S.: Geography meets Hollywood. Die Rolle der Landschaft im Spielfilm, Geogr. Z., 89, 227-236, 2001.

Fall, J.: online aufrufbar: https://www.unige.ch/sciences-societe/ geo/membres/enseignants/falljuliet/, letzter Zugriff: 18 November 2019.

FilmGeographies: online aufrufbar: https://www.filmgeographies. com/, letzter Zugriff: 11 November 2019.

Fry, P. and Thieme, S.: A social learning video method: Identifying and sharing successful transformation knowledge for sustainable soil management in Switzerland, Soil Use Manage., 35, 185194, https://doi.org/10.1111/sum.12505, 2019.

Garrett, B. L.: Videographic geographies: Using digital video for geographic research, Prog. Hum. Geog., 35, 521-541, https://doi.org/10.1177/0309132510388337, 2011.

Global Science Film Festival: online aufrufbar: https://www. sciencefilm.ch/, letzter Zugriff: 11 November 2019.

Emerging Media Lab: online aufrufbar: https://eml.ubc.ca/, letzter Zugriff: 11 November 2019.
GeoMedien Uni Kiel: online aufrufbar: https://www.geomedien. uni-kiel.de/de, letzter Zugriff: 11 November 2019.

Halffman, W. and Radder H.: The Academic Manifesto: From an Occupied to a Public University, Minerva, 53, 165-187, 2015.

Imagine Science Film Festival: online aufrufbar: http: //imaginesciencefilms.org/, letzter Zugriff: 11 November 2019.

Jacobs, J.: Listen with Your Eyes; Towards a Filmic Geography, Geography Compass, 7, 714-728, https://doi.org/10.1111/gec3.12073, 2013.

Jacobs, J.: Filmic geographies: the rise of digital film as a research method and output, Area, 48, 452-454, 2016.

JVE: online aufrufbar: http://videoethno.com/, letzter Zugriff: 11 November 2019.

Kindon, S.: Participatory video in geographic research: a feminist practice of looking?, Area, 35, 142-153, 2003.

Kindon, S.: Participatory video as a feminist practice of looking: "take two!", Area, 48, 496-503, 2016.

Labocine: online aufrufbar: https://www.labocine.com/, letzter $\mathrm{Zu}-$ griff: 11 November 2019.

mLab: online aufrufbar: https://www.geography.unibe.ch/ dienstleistungen/mlab/index_ger.html, letzter Zugriff: 11 November 2019.

Parr, H.: Collaborative film-making as process, method and text in mental health research, Cult. Geogr., 14, 114-138, 2007.

Pasquali, M.: Video in science. Protocol videos: the implications for research and society, EMBO Rep, 8, 712-716, https://doi.org/10.1038/sj.embor.7401037, 2007.

Pink, S.: Doing Visual Ethnography: Images, Media and Representation in Research, Second edition, Sage Publications, London, 2007.

Pool, G.: Der Film im Geographieunterricht, Geogr. Helv., 8, 261266, https://doi.org/10.5194/gh-8-261-1953, 1953.

Schlottmann, A. and Miggelbrink, J.: Visuelle Geographien - ein Editorial, Soc. Geogr., 4, 13-24, https://doi.org/10.5194/sg-4-132009, 2009.

Schlottmann, A., Braun, B., Bräunig, A. , and Kuhlicke, C.: Editorial, Rundbrief Geographie, 274, 1-4, 2018.

Thieme, S.: „Action“: Publishing Research Results in Film, Forum Qualitative Sozialforschung, 13, 1, https://doi.org/10.17169/FQS-13.1.1671, 2012.

Truong, J. and Schurr, C.: Global Geographies of the Intimate: New Research Group at the University of Bern, GeoAgenda, 1, 8-10, Verband Geographie Schweiz (ASG), 2019. 